\title{
Modeling the Radio and X-ray Emission of SN 1993J and SN 2002ap
}

\author{
Claes Fransson $^{1}$ and Claes-Ingvar Björnsson ${ }^{1}$ \\ Department of Astronomy, Stockholm University, AlbaNova, 10691 Stockholm, \\ Sweden \\ claes@astro.su.se, bjornsson@astro.su.se
}

Summary. Modeling of radio and X-ray observations of supernovae interacting with their circumstellar media are discussed, with special application to SN 1993J and SN 2002ap. We emphasize the importance of including all relevant physical mechanisms, especially for the modeling of the radio light curves. The different conclusions for the absorption mechanism (free-free or synchrotron self-absorption), as well as departures from an $\rho \propto r^{-2}$ CSM, as inferred by some authors, are discussed in detail. We conclude that the evidence for a variation in the mass loss rate with time is very weak. The results regarding the efficiencies of magnetic field generation and relativistic particle acceleration are summarized.

\section{Introduction}

The interaction of supernovae (SNe) with their circumstellar medium (CSM) offer important clues to both the nature of the SN progenitors, the hydrodynamics of the explosion, the environment of the SN, and the physics of high velocity shock waves. The now convincing connection of Type Ic SNe and GRB's has also made the study of the SN environment especially interesting. Basically, the standard picture of the SN interaction with the surroundings is only a non-relativistic version of the standard afterglow scenario (e.g., Chevalier, these proceedings). We will discuss a few issues related to the SN interaction. For a more detailed exposition see the recent review in [8].

\section{The Standard Model}

Supernova progenitors come in basically two flavors: extended red supergiants, or compact, hot stripped stellar cores. The former are thought to be related to Type IIP SNe, while the latter are most likely related to Type $\mathrm{Ib}$ and Ic SNe. Type IIL, IIn and IIb SNe probably represent a decreasingly massive hydrogen envelope. It is also tempting to identify this as a sequence of increasing ZAMS mass, with the Type IIP representing the most common low mass progenitors, and the Type Ic's originating from the most massive Wolf-Rayet stars. A complication is the fact that a large fraction of stars are in close binary systems. Mass transfer between the companions can in this case lead to strong mass loss even for stars of comparatively low mass. 
Massive stars have in general strong stellar winds. On the main sequence the blue supergiants have fast winds with a wind velocity of $500-3000 \mathrm{~km} \mathrm{~s}^{-1}$. As the star evolves to the red supergiant stage the escape velocity decreases dramatically and consequently the wind velocity to $10-30 \mathrm{~km} \mathrm{~s}^{-1}$. In most of this stage the mass loss rate is $10^{-6}-10^{-5} \mathrm{M}_{\odot} \mathrm{yr}^{-1}$. There are, however, indications, both from the observations of SNe (see below), and from stellar evolution calculations [14] that the star in some cases can undergo a stage with a superwind, similar to what occurs in AGB's, with mass loss rates of $\sim 10^{-4} \mathrm{M}_{\odot} \mathrm{yr}^{-1}$. The duration of this stage must be very short, $\sim 10^{4} \mathrm{yrs}$. Finally, if mass loss is important enough, the star may evolve to the WolfRayet (WR) stage, with mass loss rate $\sim 10^{-5} \mathrm{M}_{\odot} \mathrm{yr}^{-1}$ and a wind velocity of $1000-5000 \mathrm{~km} \mathrm{~s}^{-1}$, depending on the evolutionary stage of the WR star [30]. For single stars this occurs only for stars more massive than $\sim 22-40$ $\mathrm{M}_{\odot}$ for solar metallicity, depending on rotation [27]. In a binary system this may, however, occur at considerably lower mass. Because the CS density is $\rho_{\mathrm{cs}}=\dot{M} /\left(4 \pi u_{\mathrm{w}} r^{2}\right)$, where $\dot{M}$ is the mass loss rate, $u_{\mathrm{w}}$ the wind velocity and $r$ the distance from the star, the CS density into which the SN explodes can differ by several orders of magnitude, depending on the evolutionary stage in which it explodes.

The collision of the supernova ejecta with the surrounding gas generates a strong shock wave, which expands with a velocity $20-30 \%$ larger than the maximum velocity of the ejecta. The temperature behind this forward shock is $\sim 10^{9} \mathrm{~K}$. The pressure behind the shock will send a reverse shock back into the ejecta. Because of the higher density, the velocity of the reverse shock will only be $V_{\mathrm{cs}} /(n-2) \sim 500-1000 \mathrm{~km} \mathrm{~s}^{-1}$, depending on the density gradient of the ejecta, $\rho_{\mathrm{ej}} \propto r^{-n}[5]$. For polytropic envelopes the outer parts of the ejecta have $n \sim 10$ [24]. Departures from such a structure may, however, lead to both steeper and shallower gradients. For typical ejecta gradients the temperature will only be $10^{7}-10^{8} \mathrm{~K}$ behind this shock. Therefore, and because of the high ejecta density, cooling will in general be important for the reverse shock $[7,10]$. This will create a thin shell of cool gas between the reverse and CS shocks, often referred to as the cool, dense shell. The forward shock will in contrast usually be adiabatic, unless the CS density is very high.

During the last two decades radio, optical and X-ray observations have greatly added to our understanding of the SN environment (see the papers by Immler, Filippenko and Van Dyk). Of these, the radio provides the cleanest signature of CS interaction. Because of this, and also because the interpretation of these observations have generated some confusion, we will discuss these observations in some detail, as well as the complications going into the analysis.

\subsection{Radio Emission and Absorption}

The radio emission arises as a result of relativistic electrons accelerated in the shock region and emitting synchrotron radiation in the magnetic field. The 
exact mechanism of the acceleration is not well known, although a first order Fermi mechanism would be natural across the shock. Particle acceleration may, however, also occur behind the shock, close to the contact discontinuity, separating the shocked ejecta and shocked CSM. The generation of the magnetic field is not much better understood, although there has been recent progress in this area. In particular, numerical simulations [13, 29] based on ideas of Medvedev \& Loeb [26] for GRB's, have shown that the Weibel instability, which is a particular version of the two-stream instability, can give rise to a strong magnetic field at the shock. Although the simulations have mainly been done in the context of relativistic shocks, the same mechanism should work also for non-relativistic shocks. An important issue for this type of simulations is to determine the efficiency of conversion of the thermal energy of the shock into magnetic field and relativistic electron energy. The latter two are usually characterized by $\epsilon_{\mathrm{e}}=u_{\mathrm{e}} / u_{\text {therm }}$ and $\epsilon_{\mathrm{B}}=u_{\mathrm{B}} / u_{\text {therm }}$, where $u_{\text {therm }}=9 / 8 \rho V_{s}^{2}$. Most likely, these parameters depend on both the shock velocity, the CSM density and chemical composition.

The radio emission may be affected by free-free absorption from the surrounding CSM and synchrotron-self absorption (SSA) by the same relativistic electrons that emit the radiation. Assuming the relativistic electrons to be injected behind the forward shock with an energy distribution given by $d n_{\mathrm{e}}(\gamma) / d \gamma \propto \gamma^{-p}$ for $\gamma_{\min } \leq \gamma \leq \gamma_{\max }$, the synchrotron spectrum is given by $F_{\nu}=\pi R^{2} S_{\nu}\left[1-\exp \left(-\tau_{\nu}\right)\right]$ where $S_{\nu} \propto \nu^{5 / 2} / B^{1 / 2}$ and $\tau_{\nu} \propto B^{3 / 2+\alpha} N_{\mathrm{c}} \nu^{-\alpha-5 / 2}$. Here $\alpha=(p-1) / 2$ and $N_{\mathrm{e}}$ is the column density of relativistic electrons. In the case of negligible cooling $N_{\mathrm{e}} \propto R n_{\mathrm{e}} \propto R u_{\mathrm{e}}$. At low frequencies the optically thick spectrum is given by

$$
F_{\nu} \propto R^{2} \nu^{5 / 2} B^{-1 / 2}
$$

and at high frequencies

$$
F_{\nu} \propto R^{2} N_{\mathrm{e}} B^{1+\alpha} \nu^{-\alpha} \propto R^{3} n_{\mathrm{e}} B^{1+\alpha} \nu^{-\alpha} .
$$

A fit to the spectrum, covering the peak frequency $\left(\nu \equiv \nu_{\text {peak }}\right)$, therefore allows a determination of both the magnetic field and the density of relativistic electrons, if the radius of the emitting region is known. As we discuss below, this can usually be determined from optical line widths or in rare cases from VLBI.

From these expressions we can derive an estimate of the brightness temperature at the peak of the spectrum (i.e., where $\tau \sim 1$ ) given by

$$
T_{\mathrm{b}} \approx 8.2 \times \times 10^{10} g(p)^{2 / 17}\left(\epsilon_{\mathrm{e}} / \epsilon_{\mathrm{B}}\right)^{2 / 17}\left(F_{\nu} / 10^{29} \mathrm{erg} \mathrm{Hz}^{-1}\right)^{1 / 17} \mathrm{~K}
$$

independent of the value of $\nu_{\text {peak }}$. The value of $g(p)$ varies slowly with the parameters specifying the energy distribution of the relativistic electrons, for example, $g(2)=1 / \ln \left(\gamma_{\max } / \gamma_{\min }\right)$. The corresponding Lorentz factor is 


$$
\gamma_{\text {peak }} \approx 1.0 \times 10^{2}\left(\epsilon_{\mathrm{e}} / \epsilon_{\mathrm{B}}\right)^{2 / 17}\left(F_{\nu} / 10^{29} \mathrm{erg} \mathrm{Hz}{ }^{-1}\right)^{1 / 17},
$$

showing that $\gamma_{\text {peak }}$ is fairly insensitive to various parameters, and is expected to vary by less than an order of magnitude.

The second possibility, free-free absorption, is decoupled from the emission region, and only depends on the properties of the CSM. For an $\rho \propto r^{-2}$ CSM density, the free-free optical depth is

$$
\tau_{\mathrm{ff}} \propto \nu^{-2}\left(\dot{M} / u_{\mathrm{w}}\right)^{2} T^{-3 / 2} R_{\mathrm{s}}^{-3} .
$$

Here, $R_{\mathrm{s}}=V_{\mathrm{s}} t$. To estimate the relative importance of these mechanisms we determine the frequency of optical depth unity. For free-free absorption, we obtain

$$
\nu_{\mathrm{ff}} \propto\left(\dot{M} / u_{\mathrm{w}}\right) T^{-3 / 4} V_{\mathrm{s}}^{-3 / 2} t^{-3 / 2} .
$$

With

$$
B^{2} / 8 \pi \propto \epsilon_{\mathrm{B}} \rho V_{\mathrm{s}}^{2} \propto \epsilon_{\mathrm{B}}\left(\dot{M} / u_{\mathrm{w}}\right) V_{\mathrm{s}}^{2} / R_{\mathrm{s}}^{2} \propto \epsilon_{\mathrm{B}}\left(\dot{M} / u_{\mathrm{w}}\right) / t^{2}
$$

and $N_{\mathrm{e}} \propto \epsilon_{\mathrm{e}} R_{\mathrm{s}} B^{2} / \epsilon_{\mathrm{B}}$ we obtain for the SSA frequency

$$
\nu_{\mathrm{SSA}} \propto\left(\epsilon_{\mathrm{B}} \epsilon_{\mathrm{e}}\right)^{1 / 3}\left(\dot{M} / u_{\mathrm{W}}\right)^{2 / 3} V_{\mathrm{s}}^{1 / 3} t^{-1} .
$$

The ratio of these is

$$
\nu_{\mathrm{ff}} / \nu_{\mathrm{SSA}} \propto\left(\epsilon_{\mathrm{B}} \epsilon_{\mathrm{e}}\right)^{-1 / 3}\left(\dot{M} / u_{\mathrm{w}}\right)^{1 / 3} V_{\mathrm{s}}^{-11 / 3} T^{-3 / 4} t^{-1 / 2} .
$$

Therefore, the relative importance of the two absorption mechanisms depends strongly on the wind velocity of the progenitor, the efficiencies of producing relativistic electrons and the strength of the magnetic field, the CSM temperature and especially the shock velocity. The latter point has been emphasized by Chevalier [6], who find that most Type Ic SNe fall into the SSA category, because of their high expansion velocities and high wind velocities.

For a consistent modeling it is crucial to include all relevant energy loss mechanisms for the relativistic electrons. These include the usual synchrotron losses, Compton losses on the photospheric and/or the synchrotron photons, and Coulomb losses. While the former two mainly act on the high energy electrons, and steepen the spectrum, the latter is most important at low energies, and lead to a flattening of the spectrum. Even with a constant value for $p$, this will lead to a curved optically thin spectrum. In practice, the kinetic equation for the electron distribution should be solved at each energy. More details on these processes can be found in [11].

When the cooling time starts to become comparable to the dynamical time (i.e., $t$ ), radiation losses will affect the emitted synchrotron spectrum. For synchrotron cooling 


$$
t_{\text {synch }} / t=0.47(t / 10 \text { days })\left(\gamma \epsilon_{\mathrm{B}}\right)^{-1}\left(\dot{M} / 10^{-5} \mathrm{M}_{\odot} \mathrm{yr}^{-1}\right)^{-1}\left(u_{\mathrm{w}} / 10 \mathrm{kms}^{-1}\right) \text {. }
$$

Since $\gamma \gtrsim 10^{2}$, synchrotron cooling is expected to be important for supernovae with a red supergiant progenitor, unless $\epsilon_{\mathrm{B}} \ll 1$. However, cooling in supernovae with a Wolf-Rayet star progenitor cannot be excluded, since the cooling time may be shortened due to inverse Compton losses. This can occur in situations where $\epsilon_{\mathrm{e}} \gg \epsilon_{\mathrm{B}}$ (synchrotron self-Compton) or for very luminous supernovae for which scattering of photospheric photons may become important. As mentioned above, knowing $\nu_{\text {peak }}$ and $R_{\mathrm{s}}$ makes it possible to deduce individual values for $\epsilon_{\mathrm{B}}$ and $\epsilon_{\mathrm{e}}$. The occurrence of cooling provides an extra constraint on the model; for example, the underlying assumption of a spherically symmetric source geometry can be tested. The source properties can be further refined if the Compton scattered radiation is observed. Furthermore, if a low frequency flattening due to Coulomb losses is not properly accounted for, it is likely that model fitting will give a value of $\nu_{\text {peak }}$, and hence $B$, which is too large.

If free-free absorption is important, the epoch of optical depth unity allows us to determine the important density parameter, $\dot{M} / u_{\mathrm{w}}$. Because $R_{\mathrm{s}}=V_{\mathrm{s}} t$, one can determine the ratio $\dot{M} / u_{\text {w }}$ from the time of optical depth unity at a given frequency, if the shock velocity and the temperature of the CSM are known. The shock velocity can either be estimated from the maximum extent of the optical line widths, usually the $\mathrm{H} \alpha$ line, or in more rare cases, like for SN 1993J, directly from the radius as inferred from VLBI observations.

The temperature of the CSM is even more difficult to determine. At the time of shock break-out a burst of soft X-rays heats and ionizes the CSM. The spectrum and luminosity of this burst depends on the nature of the progenitor, and has recently been discussed by Matzner \& McKee [24]. A compact progenitor has a very brief burst of hard radiation with a low total energy, while a red supergiant has a longer burst, but considerably softer and with a larger energy. The ring of SN 1987A offers a spectacular example of a circumstellar structure heated and ionized by the outburst of the SN. In this case the initial temperature of the ring was $\sim 2 \times 10^{5} \mathrm{~K}$, cooling on a time scale of years $[21,22]$. At the smaller distances relevant for the free-free absorption of the radio supernovae during the first months $\left(10^{15}-10^{16} \mathrm{~cm}\right)$, these effects will be even larger.

Unfortunately, with the exception of SN 1987A, little direct information on the temperature of the CSM is available. Instead, one has to rely on theoretical calculations. Because of the low density the recombination and ionization time scales are long compared to the evolution of the ionizing spectrum and one has to calculate the evolution of the temperature and ionization of the CSM from the out-break until the epoch of interest. This has only been done for a few cases, the Type IIL SN 1979C [20], SN 1987A $[21,22]$ and SN 1993J [12]. In all cases the CSM temperatures close to the shock are $10^{5}-10^{6} \mathrm{~K}$ immediately after shock break-out. The temperature 
then decreases on a time scale of months, but can be $\gtrsim 2 \times 10^{4} \mathrm{~K}$ even a year after the explosion. This result is in sharp contrast to the simplified analyses of the radio light curves which assume a constant temperature of in most cases $\sim(1-3) \times 10^{4} \mathrm{~K}$, and obviously leads to large errors in the estimated mass loss rates. Moreover, the decreasing temperature can mimic a change in the mass loss rate with time, as has been claimed for some of the radio supernovae. These effects are discussed in detail in [20].

\section{SN 1993J}

\subsection{Synchrotron Self-absorption or Free-free Absorption?}

The radio observations of SN $1993 \mathrm{~J}[4,32,38]$ are to date by far the best ones available. Equally important, the VLBI observations [1, 23] give direct information about the size of the radio emitting region, and therefore the shock velocity, as function of time. From this combination a detailed modeling of the spectra have been possible $[11,12,31,38]$. The conclusions with regard to the mechanism behind the absorption, as well as the structure of the CSM, however, differ substantially.

The analysis in both [12] and [38] were based on modeling the radio observations by free-free absorption in a CSM. As was shown in [11], this alone cannot reproduce the turn-off of the spectrum at low frequencies, which, especially at late epochs, is considerably shallower than the exponential cutoff expected for free-free absorption. Instead, it agrees well with the standard $F_{\nu} \propto \nu^{5 / 2}$ spectrum expected from synchrotron self-absorption. Only at early epochs was free-free absorption found to be important, and then only for the longest wavelengths.

The main reason why free-free is only marginally important is that the temperature of the CSM inferred from modeling of the heating and ionization by the radiation from the shock break-out is likely to have been very high, $\sim 10^{5}-10^{6} \mathrm{~K}[12]$. The parametric fits by Van Dyk et al., on the other hand do not determine the temperature either observationally or theoretically, and simply assume a constant temperature. Because $\tau_{\mathrm{ff}} \propto T^{-3 / 2}$, this partly explains the different importance of the free-free absorption in [38] and [11]. Furthermore, Van Dyk et al. do not use the dynamical evolution from the VLBI observations. Finally, their fits do not include any cooling processes which affect the electron spectrum, and only assume a constant power law for this. Both Coulomb and synchrotron were shown to be important in [11], which flatten the low energy spectrum and steepen it at high energies. The effect of this can be seen especially in the lack of agreement between the fits and the observations at the peak of the lowest frequency, caused by Coulomb cooling, which flattens the electron spectrum.

The modeling in [31] is also based on SSA. They do, however, not include a self-consistent calculation of the electron spectrum, for cxample Coulomb 

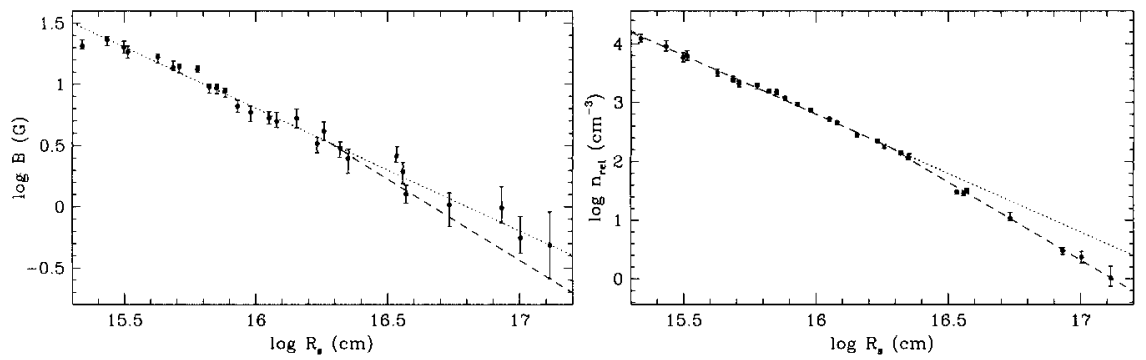

Fig. 1. Magnetic field (left) and density of relativistic electrons (right) as a function of the shock radius for SN 1993J. The dashed lines show the expected evolution if the magnetic energy density and relativistic particle density scale with the thermal energy density, $B^{2} / 8 \pi \propto \rho V_{\mathrm{s}}^{2} \propto n_{\text {rel }} \propto t^{-2}$, while the dotted lines show the case when $B \propto r^{-1}$ and $n_{\mathrm{rel}} \propto r^{-2}[11]$.

cooling is not accounted for, and invoke an arbitrary cutoff of the electron spectrum at low energies. This explains the different quantitative conclusions from [11]; in particular, the neglect of Coulomb cooling in the model fitting causes the deduced value of the synchrotron self-absorption frequency to be too high. This, in turn, results in an overestimation of the strength of the magnetic field.

A test of the SSA model was provided by the new low frequency observations with VLA in [32] and the VLA and GMRT in [4]. The fluxes of both these sets of observations agreed well with the fluxes predicted in [11]. In addition, the combined VLA and GMRT spectrum at 3200 days in [4] nicely showed the break in the spectrum caused by the synchrotron cooling to evolve as expected.

One of the most interesting results of the modeling of SN 1993J was that not only could individual values for the energy densities in magnetic field and relativistic particles be derived but also their evolution with time could be determined (see Fig. 1). Both of these energy densities scaled with the thermal energy density behind the shock (i.e., $\epsilon_{\mathrm{B}}$ and $\epsilon_{\mathrm{e}}$ are constants) but the conditions are far from equipartition since $\epsilon_{\mathrm{B}} \approx 0.14$ and $\epsilon_{\mathrm{e}} \sim 5 \times 10^{-4}$.

\subsection{X-rays}

X-ray emission from SN 1993J were observed with OSSE on Compton/GRO [18], ROSAT [15, 39], ASCA [37], Chandra [35] and XMM [40]. During the first two months, the OSSE and ASCA observations showed a very hard spectrum with $k T \sim 100 \mathrm{keV}$. This agreed well with that expected from the forward, circumstellar shock $\sim 1.1 \times 10^{2}\left(V_{\mathrm{s}} / 10^{4} \mathrm{~km} \mathrm{~s}^{-1}\right)^{2} \mathrm{keV}[12]$.

Because of the proximity to the Sun, it could then not be observed until $\sim 200$ days after explosion. The temperature was now only $\sim 1 \mathrm{keV}$, and the column density had increased by a large factor $[37,39]$. This transition 
was explained in [12] as a natural consequence of the radiative reverse shock and the presence of the cool, dense shell. As the SN expanded the column density of the CDS decreases $\propto t^{-1}$, and therefore gradually becomes optically thin. Because the luminosity below $\sim 10 \mathrm{keV}$ is dominated by the reverse shock, there will be a hard to soft transition, once the CDS becomes transparent. From the X-ray flux the mass loss rate was estimated to be $\sim 4 \times 10^{-5} \mathrm{M}_{\odot} \mathrm{yr}^{-1}$, in agreement with that estimated from the radio.

\subsection{Structure of the CSM}

There has been considerable confusion also with regard to the structure of the CSM of SN 1993J. In several papers $[12,15,36,38]$ there have been claims of a CSM density varying as $\rho \propto r^{-1.5-1.7}$. This has in turn been taken as evidence for a mass loss varying with time. Only in [11] was an $\rho \propto r^{-2}$ found to reproduce the observations. It is therefore of interest to examine the arguments on which these quite different conclusions rest.

As has already been discussed, the analysis in [12] and [38] both neglected the effects of SSA, and are therefore not physically consistent. Their conclusions from the radio modeling with regard to the CSM should therefore be ignored.

The X-ray analysis by Immler et al. [15] assumes that the X-ray emission emerges from the forward, circumstellar shock. This is directly contradicted by the low temperature, $\sim 1 \mathrm{keV}$, and high column density found from the ASCA observations later than 200 days [37]. As was discussed above, the $\mathrm{X}$-ray flux and temperature at late phases are instead consistent with those expected from the reverse shock $[12,36]$.

The analysis by Suzuki \& Nomoto [36] is based on a hydrodynamical, consistent modeling of the interaction of the ejecta and CSM, and the results should therefore be taken seriously. The fact that they obtain a CSM density at small radii varying as $\rho \propto r^{-1.7}$ and a clumpy medium at large radii, however, depends on the ejecta structure they use. The specific model they use, $4 \mathrm{H} 47$, was designed to reproduce the early light curve, and had for this purpose to be mixed artificially. More detailed modeling [16] also showed that these 1-D models are hydrodynamically unstable. A further problem with this model is that it does not reproduce the velocity evolution of the shock, as inferred by the VLBI observations. More detailed calculations of the X-ray evolution using an ejecta model which does reproduce the VLBI observations indeed show that a satisfactory reproduction of the X-ray observations can be obtained for an $\rho \propto r^{-2}$ CSM density.

In conclusion, the only self-consistent modeling of the CSM of SN 199.3J are those in [11] using radio observations and [36] using X-rays. Because of the insensitivity of the radio observations to the ejecta structure, in contrast to the X-rays, we believe the former is the more reliable, and that an $\rho \propto r^{-2}$ CSM density is the most likely. 


\section{SN 2002ap}

Type Ic SNe are of special interest because of their relation to the GRBs. The recent SN 2002ap is together with SN 1998bw the best observed objects of this class, and we discuss some issues related to this.

A special property of the Type Ic SNe is their high expansion velocities. Although this varies by a large factor from the relatively slow SN 1994I to SN 1998bw, they are in all cases much faster than the Type II's and also SN 1993J. A problem here is the fact that the expansion velocity of the radio emitting region is difficult to estimate directly from observations. The optical spectrum shows few clear line features at early time, implying that blending is very important. This only allows a lower limit to the velocity to be determined. For SN 1998bw this was $\sim 60,000 \mathrm{~km} \mathrm{~s}^{-1}[28]$, and for SN $2002 \mathrm{ap} \sim 30,000 \mathrm{~km} \mathrm{~s}^{-1}[25]$.

Because of the high expansion velocities SSA dominates the radio absorption. The radio observations of SN 1998bw [17] have been discussed in [19]. The most important conclusions was the high expansion velocity, $V_{\mathrm{s}} / c \gtrsim 0.9$, and the large energy in relativistic ejecta. Unfortunately, the radio observations of this and other Type Ic's did not allow a determination of the mass loss rate, because this depends on the unknown values of $\epsilon_{\mathrm{e}}$ and $\epsilon_{\mathrm{B}}$. While these are often assumed to be close to equipartition, there is little theoretical motivation for this.

SN 2002ap is interesting in this respect because, in addition to good radio observations [2], it was also observed with XMM [33, 34]. The X-ray emission is most naturally explained as a result of inverse Compton scattering of the photospheric radiation by the same relativistic electrons which are responsible for the radio emission [3]. This process also leads to cooling of the relativistic electrons, and thereby a steepening of the emitted spectrum, which agrees with the fact that the optically thin radio spectrum is as steep as $F_{\nu} \propto \nu^{-0.9}$.

By modeling the radio light curves of the different frequencies one can determine the expansion velocity of the radio emitting region, as well as the relative values of $\epsilon_{\mathrm{e}}$ and $\epsilon_{\mathrm{B}}$. We find that $V_{\mathrm{s}} \sim 70,000 \mathrm{~km} \mathrm{~s}^{-1}$, and that $\epsilon_{\mathrm{e}}$ and $\epsilon_{\mathrm{B}}$ are roughly equal (i.e., the magnetic field and relativistic electrons are in approximate equipartition). The exact value of their ratio depends on the upper and lower cut-offs in the electron distribution, which are not directly observable. This is in contrast to SN 1993J, where we found that $\epsilon_{\mathrm{B}} \gg \epsilon_{\mathrm{e}}$. It is also interesting to note that in both 1993J and 2002ap the injected distribution of relativistic electrons has $p \approx 2$, which is the theoretical value expected from first order Fermi-acceleration at a non-relativistic shock. This is not directly apparent from the observations, since cooling alters the emitted spectrum so that the simple relation $\alpha=(p-1) / 2$ cannot be used to deduce the value of $p$. It could be that an electron distribution with $p=2$ is valid for most supernovae and that the range of observed values for $\alpha$ is due to cooling. 


\section{Conclusions}

The VLA radio observations of SN 1993J are unique in terms of both the temporal coverage and the quality of the observations. The fact that also VLBI observations exist adds to this characterization, and means that the size of the radio emitting plasma can be determined without further assumptions. This combination makes a detailed spectral analysis possible, and from this a determination of the magnetic field and relativistic electron density as functions of time. For this a consistent physical model is necessary, which includes both the radiative transfer and the effects of different energy loss processes on the electron spectrum. There is no need to invoke arbitrary parameterized models, which only hides the real physical parameters, and may result in misleading conclusions with regard to physical mechanisms as well as the structure of the CSM.

Acknowledgement. We are grateful to Peter Lundqvist and Roger Chevalier for comments and collaborations on several of these issues.

\section{References}

1. N. Bartel et al. : Science 287, 112 (2000)

2. E. Berger, S.R. Kulkarni, R.A. Chevalier: Astrophys. J. Lett. 577, L5 (2002)

3. C.-I. Björnsson, C. Fransson: astro-ph 0401196 (2004)

4. P. Chandra, A. Ray, S. Bhatnagar: astro-ph 0402391 (2004)

5. R.A. Chevalier: Astrophys. J. 258, 790 (1982)

6. R.A. Chevalier: Astrophys. J. 499, 810 (1998)

7. R.A. Chevalier, C. Fransson: Astrophys. J. 420, 268 (1994)

8. R.A. Chevalier, C. Fransson: In: Supernovae and Gamma-Ray Bursters, LNP 598, ed. K. Weiler (Springer: Berlin 2003) p. 171

9. L. Ensman, A. Burrows: Astrophys. J. 393, 742 (1992)

10. C. Fransson: Astron. Astrophys. 133, 264 (1984)

11. C. Fransson, C.-I. Björnsson: Astrophys. J. 509, 861 (1998)

12. C. Fransson, P. Lundqvist, R.A. Chevalier: Astrophys. J. 461, 993 (1996)

13. J.T. Frederiksen, C.B. Hededal, T. Haugboelle, A. Nordlund: astro-ph 0308104 (2003)

14. A. Heger, L. Jeannin, N. Langer, I. Baraffe: Astron. Astrophys. 327, 224 (1997)

15. S. Immler, B. Aschenbach, Q.D. Wang: Astrophys. J. Lett. 561, L107 (2001)

16. K. Iwamoto, T.R. Young, N. Nakasato, T. Shigeyama, K. Nomoto, I. Hachisu, H. Saio: Astrophys. J. 477, 865 (1997)

17. S.R. Kulkarni et al. : Nature 395, 663 (1998)

18. M.D. Leising et al. : Astrophys. J. Lett. 431, L95 (1994)

19. Z. Li, R.A. Chevalier: Astrophys. J. 526, 716 (1999)

20. P. Lundqvist, C. Fransson: Astron. Astrophys. 192, 221 (1988)

21. P. Lundqvist, C. Fransson: Astrophys. J. 380, 575 (1991)

22. P. Lundqvist, C. Fransson: Astrophys. J. 464, 924 (1996)

23. J.M. Marcaide et al. : Astrophys. J. Lett. 486, L31 (1997) 
24. C.D. Matzner, C.F. McKee: Astrophys. J. 510, 379 (1999)

25. P.A. Mazzali et al. : Astrophys. J. Lett. 572, L61 (2002)

26. M.V. Medvedev, A. Loeb: Astrophys. J. 526, 697 (1999)

27. G. Meynet, A. Maeder: Astron. Astrophys. 404, 975 (2003)

28. T. Nakamura, P.A. Mazzali, K. Nomoto, K. Iwamoto: Astrophys. J. 550, $991(2001)$

29. K.-I. Nishikawa, P. Hardec, G. Richardson, R. Preece, H. Sol, G.J. Fishman: astro-ph 0305091 (2003)

30. T. Nugis, H.J.G.L.M. Lamers: Astron. Astrophys. 360, 227 (2000)

31. M.A. Pérez-Torres, A. Alberdi, J.M. Marcaide: Astron. Astrophys. 374, 997 (2001)

32. M.A. Pérez-Torres, A. Alberdi, J.M. Marcaide: Astron. Astrophys. 394, $71(2002)$

33. P. Soria, E. Pian, P. Mazzali: Astron. Astrophys. 413, 107 (2004)

34. F.K. Sutaria, P. Chandra, S. Bhatnagar, A. Ray: Astron. Astrophys. 397, $1011(2003)$

35. D.A. Swartz, K.K. Ghosh, M.L. McCollough, T.G. Pannuti, A.F. Tennant, K. Wu: Astrophys. J. Suppl. 144, 213 (2003)

36. T. Suzuki, K. Nomoto: Astrophys. J. 455, 658 (1995)

37. S. Uno et al. : Astrophys. J. 565, 419 (2002)

38. S.D. Van Dyk, K.W. Weiler, R.A. Sramek, M.P. Rupen, N. Panagia: Astrophys. J. Lett. 432, L115 (1994)

39. H.U. Zimmermann et al. : Nature 367, 621 (1994)

40. H.U. Zimmermann, B. Aschenbach: Astron. Astrophys. 406, 969 (2003) 